\title{
3 Analytical estimates of the pull-in voltage for carbon 4 nanotubes considering tip-charge concentration 5 and intermolecular forces
}

\author{
6 Giovanni Bianchi $\cdot$ Enrico Radi [
}

7 Received: 9 July 2019/Accepted: 28 December 2019

8 (c) Springer Nature B.V. 2020

9 Abstract Two-side accurate analytical estimates of 10 the pull-in parameters of a carbon nanotube switch 11 clamped at one end under electrostatic actuation are 12 provided by considering the proper expressions of the 13 electrostatic force and van der Waals interactions for a 14 carbon nanotube, as well as the contribution of the 1. $\mathbf{A Q 1}$ charge concentration at the free end. According to the 16 Euler-Bernoulli beam theory, the problem is governed 17 by a fourth-order nonlinear boundary value problem. 18 Two-side estimates on the centreline deflection are 19 derived. Then, very accurate lower and upper bounds 20 to the pull-in voltage and deflection are obtained as 2 AQ2 function of the geometrical and material parameters. 22 The analytical predictions are found to agree remark23 ably well with the numerical results provided by the 24 shooting method, thus validating the proposed 25 approach. Finally, a simple closed-form relation is 26 proposed for the minimum feasible gap and maximum 27 realizable length for a freestanding CNT cantilever.

\footnotetext{
A1 G. Bianchi · E. Radi $(\square)$

A2 Dipartimento di Scienze e Metodi dell'Ingegneria,

A3 Università di Modena e Reggio Emilia, Via G. Amendola

A4 2, 42122 Reggio Emilia, Italy

A5 e-mail: enrico.radi@unimore.it

A6 E. Radi

A7 Centro Interdipartimentale "En\&Tech", Via G.

A8 Amendola, 2, 42122 Reggio Emilia, Italy
}

Keywords Carbon nanotube $\cdot$ Pull-in voltage

NEMS · Nanocantilever - van der Waals interactions · Charge concentration

\section{Introduction}

Carbon nanotubes (CNTs) display a number of smart electronic and mechanical properties that are currently exploited in a wide variety of industrial applications, such as sensors, nanoactuators, memory devices, switches, high frequency nanoresonators and nanotweezers [1-3]. Due to their tiny size, CNTs display ultra-low mass and very high resonance frequency. Moreover, they undergo purely elastic behaviour, they are able to carry huge electrical currents and to sustain high current densities. These attractive properties, in conjunction with the significant progress recently made in the fabrication of carbon nanostructures, allow CNTs to become essential components in the production of enhanced nano-electro-mechanical systems (NEMS) [1]. As a consequence, a considerable amount of research interest has been dedicated to the accurate modelling of the structural and electric behavior of CNTs in the last few years.

A typical CNT switch consists in a moveable nanowire suspended over a fixed conductive ground plane, usually made of graphite. By applying DC voltage difference between the components, the CNT

\begin{tabular}{|l|lll|}
\hline & Journal : Medium 11012 & Dispatch : 9-1-2020 & Pages : 17 \\
Article No. : $\mathbf{1 1 1 9}$ & $\square$ LE & $\square$ TYPESET \\
MS Code : MECC-D-19-00479R2 & $\checkmark$ CP & $\checkmark$ DISK \\
\hline
\end{tabular}


deflects toward the ground electrode until at the pull-in voltage it sticks on the ground plane, thus shortening the electric circuit. The atomic interactions at the nano-scale separations, modelled by the van der Waals force, substantially affects the pull-in instability of NEMS. Both the electrostatic and van der Waals forces depend on the CNT deflection non-linearly. This occurrence makes every attempt to describe their response in closed form a very difficult task. Specifically, no exact solution can be found for the nonlinear ordinary differential equation (ODE) governing the CNT deflection under electrostatic actuation. As a consequence, a variety of numerical and approximated approaches has been proposed in the technical literature, ranging from the reduction to 1D lumped models, based on the assumption of appropriate shape functions for the CNT deflection, to the use of powerful numerical techniques to generate reducedorder models, such as the Differential Quadrature Method, the Galerkin Discretization Method or the Finite Element Method [4-11]. However, these approximated methods may provide significant error percentages as the CNT deflection increases and gets closer to the pull-in limit. Moreover, they predict arbitrary estimates of the effective pull-in parameters, whereas an effective approach should provide accurate lower and upper bounds that can be exploited for ensuring the safe operation of the device. Alternatively, molecular dynamics approaches have been adopted to study CNTs pull-in behavior [12]. However, these methods are very time-consuming and can not be easily employed for large structures.

As remarked by Ke et al. [13, 14], electric charges tend to concentrate at the ends of a linear conductor and thus for proper modeling of the pull-in instability phenomenon the effect of the concentrated load due to charge concentration at the end of a CNT cantilever is expected to provide a significant contribution on the deflection of CNT and consequently on the pull-in instability. Therefore, it must be necessarily considered for the accurate evaluation of the pull-in voltage. In particular, Ke et al. [13] showed that the pull-in voltage decreases by about $14 \%$ due to the effect of the tip-charge concentration. They also provided an approximate relation for the pull-in voltage that account for the effects of tip-charge concentration and finite kinematics. They found that the finite kinematic effect is negligible for a CNT-based cantilever switch, but the effect of charge concentration is quite significant. Ke [15] also presented a detailed review of the recent advances in the electro-mechanical modeling and characterization of CNT cantilevers and their applications.

The development of analytical models that can predict the pull-in response of the device becomes extremely relevant for identifying the most efficient geometries and materials required for meeting the requests of ultralow power consumption, strength and durability. Despite the amount of numerical and approximated investigations, analytical models and closed form expressions for assessing the occurring of CNT pull-in instability still appears to be limited. An accurate determination of the stable actuating range and the pull-in instability threshold is a crucial issues for the design of reliable and optimized CNT-based NEMS. In two previous works, Radi et al. [16, 17] provided an analytical methodology for assessing accurate lower and upper bounds to the pull-in parameters of an electro-statically actuated micro- or nano-cantilever, by taking the contributions of flexible support and compressive axial load into consideration. Both contributions are found to reduce the pull-in voltage and to increase the critical gap spacing for a freestanding nano-cantilever, namely in the absence of electrical actuation. The investigations [16, 17] have focused on the pull-in instability in micro- and nanobeams with rectangular cross-section only. Moreover, the contribution of the charge concentrated at the nanocantilever tip has been neglected in these works.

In the present work, attention is paid to investigate the pull-in phenomenon in CNT with circular crosssection rolled up by graphene sheets, by considering the proper expressions of the electrostatic force as well as the significant effect induced by the tip-charge concentration [13, 14, 18, 19]. The van der Waals force acting on the CNT has been derived in [4] starting from the Lennard-Jones potential (see also [8, 20-22]). The finite kinematic effect has been neglected here, Ke et al. [13, 14] found indeed that for a clamped CNT it becomes significant only for very slender CNTs and large gap spacing. Indeed, the pullin instability generally occurs as the CNT tip deflections attains about $1 / 3 \div 1 / 2$ of the gap spacing, which is much smaller than the CNT length. Within this range, the CNT can be reasonably supposed to experience small deformations and small displacement. Therefore, reference is made here to the classic Euler-Bernoulli (EB) beam theory, which is valid for

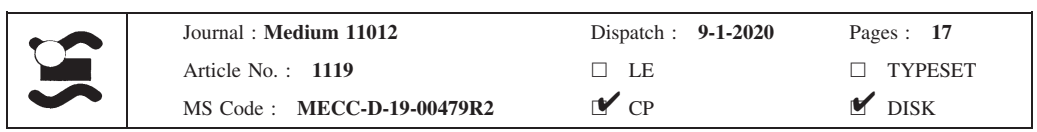


most of the CNT applications as switches and actuators [23]. The main advantage of the present approach with respect to other ones proposed in literature consists in providing accurate analytical bounds from above and below for the pull-in voltage and pull-in deflection, thus avoiding the numerical integration of the nonlinear fourth-order ODE derived from the EB beam theory. Moreover, the present work extends previous investigations on nanobeams with rectangular cross section [16, 17], which are not specifically addressed to CNTs and do not take the contribution of the concentrated-tip charge into account.

By introducing few non-dimensional parameters, the nonlinear ODE for the CNT centreline deflection and the corresponding boundary conditions are presented in Sect. 2. Moreover, an equivalent integral equation formulation is derived therein. The nonlinear response is due to the electrostatic force and van der Waals interactions, which depends on the beam deflection nonlinearly, whereas the CNT is modelled by using a linear elastic EB beam. The solution of the boundary value problem is then proved to be positive, increasing and convex. Upper and lower estimates for the CNT deflection are obtained in Sect. 3. Accurate two-side analytical bounds to the pull-in parameters are derived in Sect. 4 by exploiting the estimates obtained in Sect. 3. The accuracy of the proposed bounds are then validated in Sect. 5 by comparing the analytical estimates and the numerical results provided by the shooting method. A remarkable agreement is observed therein. On the basis of the obtained results, an approximated closed-form expression is finally proposed for permissible gap spacing and CNT length under the influence of intermolecular 186 attractions.

The approach here proposed refers to a singlewalled CNT. However, it can be easily generalized to multi-walled CNTs, e.g. by considering the expressions of the electrostatic and van der Waals forces provided in [24], as well as to other kinds of interactions, such as capillary and electrochemical forces $[25,26]$.

\section{Mathematical modeling}

A schematic view of a CNT-based cantilever switch is shown in Fig. 1. A movable single-walled or multiwalled CNT is placed above a fixed ground plane and subject to van der Waals interactions and attractive electrostatic force due to applied voltage. The nanotube length and the cross section mean radius are denoted with $L$ and $R$, respectively. The gap spacing between the nanotube and the ground plane is denoted by $H$. The deflection $v(z)$ of the CNT centreline is described by the following non-linear fourth-order ODE written in terms of the nondimensional variables $u=v / H$ and $x=z / L$ for $0 \leq x \leq 1$ and $0 \leq u \leq 1$

$u^{\mathrm{IV}}(x)=f(u(x)), \quad$ for $\quad x \in[0,1]$,

where the prime denotes differentiation with respect to the function argument. The CNT actuation is modelled by considering both contributions of electrostatic force and van der Waals interactions, namely
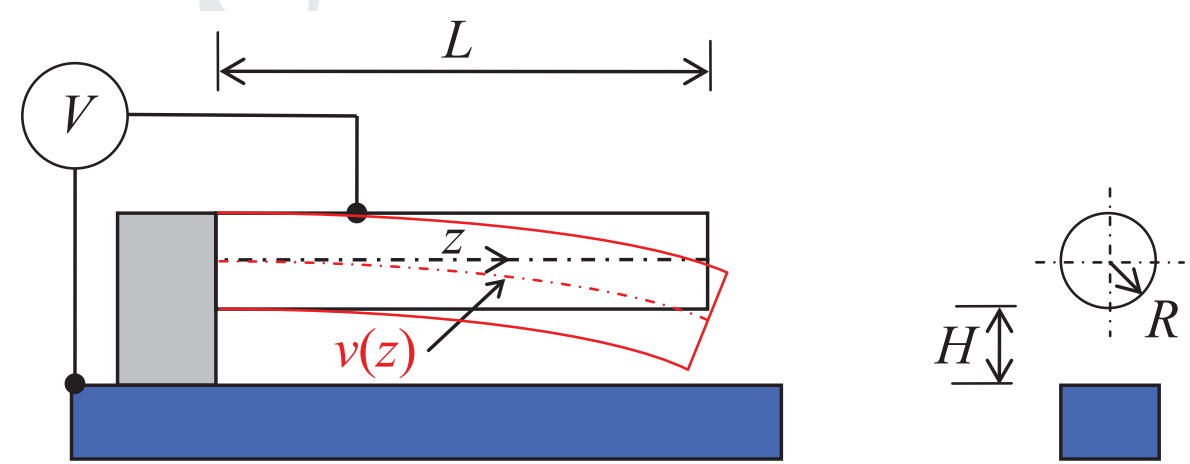

Fig. 1 A CNT based cantilever switch under electrostatic loading

\begin{tabular}{|l|ll|} 
Journal : Medium 11012 & Dispatch : 9-1-2020 & Pages : 17 \\
Article No. : 1119 & $\square$ LE & $\square$ TYPESET \\
MS Code : MECC-D-19-00479R2 & $\checkmark$ CP & $\checkmark$ DISK \\
\hline
\end{tabular}


$f(u)=\beta f_{e}(u)+\gamma F_{c}(u)$, where the normalized electrostatic and van der Waals forces for the cylindrical geometry are given by $[4,8,13,14,18-22,27,28]$

$$
\begin{aligned}
f_{e}(u) & =\frac{1}{\sqrt{(1-u)(1-u+2 / k)}\left[\cosh ^{-1}(1+k-k u)\right]^{2}}, \\
f_{c}(u) & =\frac{8 k^{4}(1-u)^{4}+32 k^{3}(1-u)^{3}+72 k^{2}(1-u)^{2}+80 k(1-u)+35}{k^{10}[(1-u)(1-u+2 / k)]^{9 / 2}},
\end{aligned}
$$

where $k=H / R$ is a geometric ratio and the nondimensional parameters $\beta$ and $\gamma$ are proportional to the magnitude of the electrostatic force and van der Waals interactions, respectively, namely

$\beta=\frac{\pi \varepsilon_{0} V^{2} L^{4}}{H^{2} E I}, \quad \gamma=\frac{C_{6} \sigma^{2} \pi^{2} L^{4}}{2 R^{5} E I}$

where $\varepsilon_{0}=8.854 \times 10^{-12} \mathrm{C}^{2} \mathrm{~N}^{-1} \mathrm{~m}^{-2}$ is the permittivity of vacuum, $V$ is the electric voltage applied to the electrodes, $C_{6}=15.2 \mathrm{Ev} \AA^{6}$ is a constant characterizing the interaction between carbon-carbon atoms, $\sigma=38 \mathrm{~nm}^{-2}$ is the graphene surface density, $I \approx \pi t$
$R^{3}$ is the moment of inertia of the CNT cross-section, where $t$ is the CNT wall thickness, and $E$ is the Young's modulus of the graphene. A number of studies based on experimental tests and atomistic simulations found that the Young's modulus of the graphene varies from 0.5 to $5.5 \mathrm{TPa}$ and the single wall thickness ranges between 0.7 and $3.4 \AA$, see the summary of results given in [29]. The mean values suggested in [29] are $t=1.34 \AA$ and $E=2.52 \mathrm{TPa}$. been derived in [4] by taking the derivative with respect to the deflection of the van der Waals energy determined by double volume integral of the LennardJones potential.

The boundary conditions for the cantilever EB beam then require vanishing of displacement and
The van der Waals force per unit length $(3)_{2}$ has rotation at the clamped end $(x=0)$, vanishing of the bending moment and assigned shearing force at the free end $(x=1)$, namely

$u(0)=0, \quad u^{\prime}(0)=0, \quad u^{\prime \prime}(1)=0$,
$u^{\prime \prime \prime}(1)=-\beta q(\delta)$,

where $\delta=u(1)$ is the tip displacement and

$q(\delta)=\frac{0.85 \rho(1+k)^{2 / 3}}{\sqrt{(1-\delta)(1-\delta+2 / k)}\left[\cosh ^{-1}(1+k-k \delta)\right]^{2}}$,

is the normalized shearing force due to the electrostatic attraction of the charge concentrated at the CNT tip [13, 14, 27], being $\rho=R / L$ the inverse of the CNT slenderness.

By taking the derivative of Eq. (2) with respect to $u$, one obtains

$f^{\prime}(u)=\beta f_{e}^{\prime}(u)+\gamma F_{c}^{\prime}(u)$,

where
Note that the functions $f(u)$ and $f^{\prime}(u)$ defined in (2) and (7) are positive and monotonically increasing for $0 \leq u \leq 1$ and $k>0$, namely

$f(u) \geq f(0) \geq 0, \quad f^{\prime}(u) \geq f^{\prime}(0) \geq 0$,

where 8

$$
\begin{aligned}
f(0)= & \frac{\beta}{\sqrt{(1+2 / k)}\left[\cosh ^{-1}(1+k)\right]^{2}} \\
& +\gamma \frac{8 k^{4}+32 k^{3}+72 k^{2}+80 k+35}{k^{10}(1+2 / k)^{9 / 2}},
\end{aligned}
$$

\begin{tabular}{|l|ll|}
\hline Journal : Medium 11012 & Dispatch : 9-1-2020 & Pages : 17 \\
Article No. : $\mathbf{1 1 1 9}$ & $\square$ LE & $\square$ TYPESET \\
MS Code : MECC-D-19-00479R2 & $\checkmark_{\mathrm{CP}}$ & $\checkmark$ DISK \\
\hline
\end{tabular}




$$
\begin{aligned}
f^{\prime}(0)= & \beta \frac{2 \sqrt{k(2+k)}+(1+k) \cosh ^{-1}(1+k)}{(2+k) \sqrt{(1+2 / k)}\left[\cosh ^{-1}(1+k)\right]^{3}} \\
& +5 \gamma \frac{8 k^{5}+40 k^{4}+120 k^{3}+200 k^{2}+175 k+63}{k^{11}(1+2 / k)^{11 / 2}} .
\end{aligned}
$$

2672.1 Nonlinear integral equation for $u(x)$

268

269

270

271

272
In this section, the governing ODE (1) is integrated four times by using the boundary conditions (5), in order to obtain preliminary estimates about the solution $u(x)$ and its derivatives up to the third order. Moreover, a nonlinear integral equation for the deflection $u$ is obtained, which will be used later for achieving accurate bounds for the pull-in parameters. A first integration of the governing ODE (1) between $x$ and 1 by using the boundary condition $(5)_{4}$ yields

$u^{\prime \prime \prime}(x)=-\int_{x}^{1} f(u(t)) d t-\beta q(\delta)$.

Integration of Eq. (12) between $x$ and 1, by using the boundary condition $(5)_{3}$ and integration by parts, then yields

$u^{\prime \prime}(x)=\int_{x}^{1}(t-x) f(u(t)) d t+(1-x) \beta q(\delta)$.

Integration of Eq. (13) between 0 and $x$, by using the boundary condition $(5)_{2}$ and integration by parts, then yields

$$
\begin{aligned}
u^{\prime}(x)= & \frac{1}{2}\left\{x \int_{x}^{1}(2 t-x) f(u(t)) d t+\int_{0}^{x} t^{2} f(u(t)) d t\right. \\
& +\beta q(\delta)(2-x) x\} .
\end{aligned}
$$

Finally, integration of Eq. (14) between 0 and $x$ by using the boundary condition $(5)_{1}$ and integration by parts gives the following nonlinear integral equation for $u(x)$

$$
\begin{aligned}
u(x)= & \frac{1}{6}\left\{x^{2} \int_{x}^{1}(3 t-x) f(u(t)) d t\right. \\
& \left.+\int_{0}^{x}(3 x-t) t^{2} f(u(t)) d t+\beta q(\delta)(3-x) x^{2}\right\} .
\end{aligned}
$$

The normalized deflection of the cantilever tip, $\delta=u(1)$, then must satisfy the following condition derived from Eq. (15) for $x=1$

$\delta=\frac{1}{6} \int_{0}^{1}(3-t) t^{2} f(u(t)) d t+\frac{1}{3} \beta q(\delta)$.

Considering that $f(u) \geq 0$ and $q(\delta) \geq 0$, from Eqs. (1), 295 (12)-(15) the following conditions then hold true for 296 $x \in[0,1]:$

$$
\begin{aligned}
& u(x) \geq \frac{x^{2}}{6}(3-x) \beta q(\delta) \geq 0, \\
& u^{\prime}(x) \geq\left(x-\frac{x^{2}}{2}\right) \beta q(\delta) \geq 0 \\
& u^{\prime \prime}(x) \geq(1-x) \beta q(\delta) \geq 0 \\
& u^{\prime \prime \prime}(x) \leq-\beta q(\delta)
\end{aligned}
$$

Therefore, the function $u(x)$ is positive, increasing and convex for $x \in(0,1)$.

\section{Two-side estimates for the deflection}

In order to define upper and lower bounds to the pull-in parameters, two-side estimates are first derived for the deflection $u(x)$.

3.1 Upper bounds to the deflection $u(x)$

Let $u(x)$ denotes the solution to the BVP (1) and (5), 306 then it can be proved that $u(x) \leq u_{U}(x)$ for $x \in[0,1], \quad 307$ where

$u_{U}(x)=\delta b_{1}(x)+\beta q(\delta)\left[b_{2}(x)+f^{\prime}(0) b_{3}(x)\right]$,

and

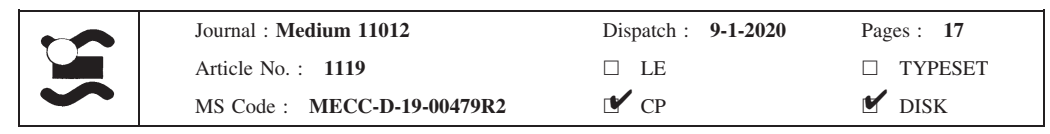




$$
\begin{aligned}
& b_{1}(x)=\frac{1}{3} x^{2}\left(x^{2}-4 x+6\right) \geq 0, \\
& b_{2}(x)=\frac{1}{18} x^{2}(1-x)(2 x-3) \geq 0,
\end{aligned}
$$

$$
b_{3}(x)=\frac{1}{5040} x^{2}(1-x)\left(x^{4}-6 x^{3}-6 x^{2}+38 x-33\right) \leq 0 \text {. }
$$

314 Indeed, let us define the function

$h(x)=\delta b_{1}(x)+\beta q(\delta)\left[b_{2}(x)+f^{\prime}(0) b_{3}(x)\right]-u(x)$,

then the derivatives of $h(x)$ up to the fourth order become
Therefore, the function $h(x)$ satisfies all the requirements for the application of Lemma A reported in the "Appendix", and thus $h(x) \geq 0$ for $x \in[0,1]$, so that, by using the definition (21), the upper bound (18) for the CNT deflection holds true.

The term $\delta b_{1}(x)$ appearing in (18) coincides with the quartic polynomial used for approximating nanobeam deflection in [30]. Moreover, from conditions (18), by using $(9)_{2}$ and (20) it follows that $u(x) \leq \delta b_{1}(x)+\beta q(\delta) b_{2}(x), \quad$ for $\quad x \in[0,1]$,

Obviously, the upper bound (25) is less accurate than (18), but it depends linearly on the parameter $\beta$.

$$
\begin{aligned}
& h^{\prime}(x)=4 \delta\left(\frac{x^{3}}{3}-x^{2}+x\right)-\frac{\beta}{18} q(\delta)\left[\frac{f^{\prime}(0)}{280}\left(7 x^{6}-42 x^{5}+176 x^{3}-213 x^{2}+66 x\right)+8 x^{3}-15 x^{2}+6 x\right]-u^{\prime}(x), \\
& h^{\prime \prime}(x)=4 \delta(1-x)^{2}+\frac{\beta}{3} q(\delta)(1-x)\left[\frac{f^{\prime}(0)}{280}\left(7 x^{4}-28 x^{3}-28 x^{2}+60 x-11\right)+4 x-1\right]-u^{\prime \prime}(x), \\
& h^{\prime \prime \prime}(x)=-8 \delta(1-x)-\frac{\beta}{3} q(\delta)\left[\frac{f^{\prime}(0)}{280}\left(35 x^{4}-140 x^{3}+176 x-71\right)+8 x-5\right]-u^{\prime \prime \prime}(x), \\
& h^{\mathrm{IV}}(x)=8 \delta-\frac{\beta}{3} q(\delta)\left[\frac{f^{\prime}(0)}{70}\left(35 x^{3}-105 x^{2}+44\right)+8\right]-u^{\mathrm{IV}}(x) .
\end{aligned}
$$

Moreover, by taking the derivative of $h^{\mathrm{IV}}(x)$, using Eq. (1), one has

$$
h^{\mathrm{V}}(x)=\beta q(\delta) f^{\prime}(0)\left(x-\frac{1}{2} x^{3}\right)-f^{\prime}(u) u^{\prime}(x) \leq 0,
$$

where the last inequality follows from $(9)_{2}$ and $(17)_{2}$, thus implying that the function $h^{\prime \prime \prime}(x)$ is concave. Then, the following conditions are met by function $h(x)$ and its derivatives

$$
\begin{aligned}
& h(0)=0, \quad h(1)=0, \quad h^{\prime}(0)=0, \quad h^{\prime \prime}(1)=0, \\
& h^{\prime \prime \prime}(1)=0, \quad h^{\mathrm{V}}(x) \leq 0 .
\end{aligned}
$$

Therefore, two slightly different procedures for deriving lower bound to the pull-in parameters will be developed in Sect. 4.1 starting from the bounds (18) and (25), respectively.

\subsection{Lower bounds to the deflection $u(x)$}

Let $u(x)$ denote the solution to the BVP (1) and (5), 344 then the lower bound $u(x) \geq u_{L}(x)$ holds true for 345 $x \in[0,1]$, where

$u_{L}(x)=\delta a_{1}(x)+f(0) a_{2}(x)$

and

$$
\begin{aligned}
& a_{1}(x)=\frac{1}{2}\left(3 x^{2}-x^{3}\right) \geq 0, \\
& a_{2}(x)=\frac{1}{48}\left(3 x^{2}-5 x^{3}+2 x^{4}\right) \geq 0 .
\end{aligned}
$$

Let us indeed define the following function 46

\section{8} . 


$$
\begin{aligned}
g(x)= & u(x)-\frac{\delta}{2}\left(3 x^{2}-x^{3}\right) \\
& -\frac{f(0)}{48}\left(3 x^{2}-5 x^{3}+2 x^{4}\right),
\end{aligned}
$$

then the derivatives of $g(x)$ write

$$
\begin{aligned}
g^{\prime}(x) & =u^{\prime}(x)-\frac{3}{2} \delta\left(2 x-x^{2}\right)-\frac{f(0)}{48}\left(6 x-15 x^{2}+8 x^{3}\right), \\
g^{\prime \prime}(x) & =u^{\prime \prime}(x)-3 \delta(1-x)-\frac{f(0)}{8}\left(1-5 x+4 x^{2}\right), \\
g^{\prime \prime \prime}(x) & =u^{\prime \prime \prime}(x)+3 \delta+(5-8 x), \\
g^{\mathrm{IV}}(x) & =u^{\mathrm{IV}}(x)-f(0) \geq 0,
\end{aligned}
$$

where the latter inequality follows from Eqs. (1) and $(9)_{1}$. Therefore, the function $g(x)$ satisfies the following boundary conditions

$g(0)=0, \quad g(1)=0, \quad g^{\prime}(0)=0, \quad g^{\prime \prime}(1)=0$.

\section{Bounds to the pull-in parameters}

By introducing the estimates (18), (25) and (26) on the deflection $u(x)$ in relation (16), the following lower and upper bounds to the pull-in parameters $\beta_{P I}$ and $\delta_{P I}$ can be derived analytically.

\subsection{Accurate lower bounds to the pull-in parameters}

By using $(9)_{2}$ and the upper bound to the CNT deflection (18) one has $f(u) \leq f\left(u_{U}\right)$, then from (16) it follows

$$
\delta \leq F(\delta, \beta)+\frac{\beta}{3} q(\delta),
$$

374 where the function

$$
F(\delta, \beta)=\frac{1}{6} \int_{0}^{1} t^{2}(3-t) f\left(u_{U}(t)\right) d t
$$

can be calculated numerically. $\quad 376$

Condition (31) defines a lower bound to the relation $\quad 377$ between the electrostatic loading parameter $\beta$ and the 378 normalized pull-in deflection $\delta$. The maximum value $\quad 379$ of the parameter $\beta$ and the corresponding tip deflection $\quad 380$ $\delta$ obtained from relation (31) by using the stationary 381 condition

$\frac{\partial \beta}{\partial \delta}=0$

then define the lower bounds of the pull-in parameters $\beta_{L}$ and $\delta_{L}$, such that $\beta_{P I} \geq \beta_{L}$ and $\delta_{P I} \geq \delta_{L}$, which are given by the following two conditions

$$
\begin{gathered}
F\left(\delta_{L}, \beta_{L}\right)+\frac{\beta_{L}}{3} q\left(\delta_{L}\right)=\delta_{L}, \\
\Phi\left(\delta_{L}, \beta_{L}\right)+\frac{\beta_{L}}{3} q^{\prime}\left(\delta_{L}\right)=1,
\end{gathered}
$$

where the function

$$
\begin{aligned}
\Phi(\delta, \beta)= & \frac{1}{6} \int_{0}^{1} t^{2}(3-t)\left\{b_{1}(t)+\beta q^{\prime}(\delta)\left[b_{2}(t)\right.\right. \\
& \left.\left.+f^{\prime}(0) b_{3}(t)\right]\right\} f^{\prime}\left(u_{U}(t)\right) d t
\end{aligned}
$$

can be calculated numerically and is given by the derivative with respect to $\delta$ of the function $F(\delta, \beta)$ defined in (32), performed by considering the maximization condition (33) and the definition (18) of $u_{U}$.

\subsubsection{Lower bounds to the pull-in parameters}

By using the estimate (25) and the monotony condi395 tions $f_{e}^{\prime}(u) \geq 0$ and $f_{c}^{\prime}(u) \geq 0$, from (16) it follows

$\delta \leq \beta f_{e}(\delta)+\gamma F_{c}(\delta)+\frac{\beta}{3} q(\delta)$,

where the functions

$F_{e}(\delta)=\frac{1}{6} \int_{0}^{1} t^{2}(3-t) f_{e}\left(\delta b_{1}(t)-\beta q(\delta) b_{2}(t)\right) d t$

$$
F_{c}(\delta)=\frac{1}{6} \int_{0}^{1} t^{2}(3-t) f_{c}\left(\delta b_{1}(t)-\beta q(\delta) b_{2}(t)\right) d t
$$

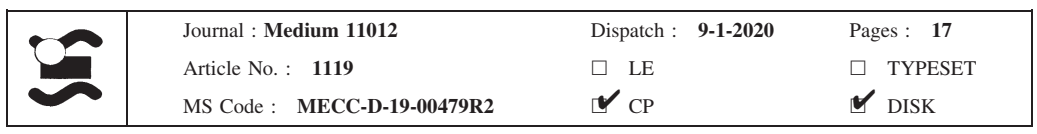


402

403

404

405

406

407

408

409 can be calculated numerically.

Condition (36) defines a lower bound to the relation between the electrostatic loading parameter $\beta$ and the normalized pull-in deflection $\delta$. In this case, inequality (36) can be easily solved for the parameter $\beta$. The maximum value of the parameter $\beta$ and the corresponding tip deflection $\delta$ obtained from relation (36) by using the stationary condition (33) then provides the lower bounds of the pull-in parameters $\beta_{L}$ and $\delta_{L}$. Namely, the latter values are given by the conditions

$$
\begin{gathered}
\beta_{L} F_{e}\left(\delta_{L}\right)+\gamma F_{c}\left(\delta_{L}\right)+\frac{\beta_{L}}{3} q\left(\delta_{L}\right)=\delta_{L}, \\
\beta_{L} F_{e}^{\prime}\left(\delta_{L}\right)+\gamma F_{c}^{\prime}\left(\delta_{L}\right)+\frac{\beta_{L}}{3} q^{\prime}\left(\delta_{L}\right)=1,
\end{gathered}
$$

where the apex denotes the derivative with respect to the function argument within the brackets, namely

$$
\begin{aligned}
F_{e}^{\prime}(\delta)= & \frac{1}{6} \int_{0}^{1} t^{2}(3-t)\left[b_{1}(t)-\beta q^{\prime}(\delta) b_{2}(t)\right] f_{e}^{\prime}\left(\delta b_{1}(t)\right. \\
& \left.-\beta q(\delta) b_{2}(t)\right) d t,
\end{aligned}
$$

$$
\begin{aligned}
F_{c}^{\prime}(\delta) & =\frac{1}{6} \int_{0}^{1} t^{2}(3-t)\left[b_{1}(t)-\beta q^{\prime}(\delta) b_{2}(t)\right] f_{c}^{\prime}\left(\delta b_{1}(t)\right. \\
& \left.-\beta q(\delta) b_{2}(t)\right) d t
\end{aligned}
$$

The latter functions can be calculated numerically and are given by the derivative with respect to $\delta$ of the functions defined in (37) and (38), performed by considering the maximization condition (33).

\subsection{Upper bounds to the pull-in parameters}

By using $(9)_{2}$ and the lower bound to the CNT deflection (26) it follows that $f(u) \geq f\left(u_{L}\right)$, then from (16) one has

$\delta \geq G(\delta, \beta)+\frac{\beta}{3} q(\delta)$,

where the function

$G(\delta, \beta)=\frac{1}{6} \int_{0}^{1} t^{2}(3-t) f\left(u_{L}(x)\right) d t$ can be calculated numerically.

Inequality (42) implicitly defines an upper bound to the relation between the parameters $\beta$ and $\delta$. The maximum value of the parameters $\beta$ and the corresponding tip deflection $\delta$ obtained from this relation by using the stationary condition (33) then provides the upper bounds of the pull-in parameters $\beta_{U}$ and $\delta_{U}$, such that $\beta_{P I} \leq \beta_{U}$ and $\delta_{P I} \leq \delta_{U}$. Therefore, the upper bounds follow from the two conditions

$$
\begin{array}{r}
G\left(\delta_{U}, \beta_{U}\right)+\frac{\beta_{U}}{3} q\left(\delta_{U}\right)=\delta_{U}, \\
\Gamma\left(\delta_{U}, \beta_{U}\right)+\frac{\beta_{U}}{3} q^{\prime}\left(\delta_{U}\right)=1,
\end{array}
$$

where the function

$$
\Gamma(\delta, \beta)=\frac{1}{6} \int_{0}^{1} t^{2}(3-t) a_{1}(t) f^{\prime}\left(u_{L}(x)\right) d t,
$$

can be calculated numerically and is given by the derivative with respect to $\delta$ of the function $G(\delta, \beta)$ defined in (43), performed by considering the maximization condition (33) and the definition (26) of $u_{L}$.

4.3 Ke et al. estimates to the pull-in voltage

The following approximated relation for the pull-in voltage of a CNT whose radius $R$ is much smaller than the gap spacing $H$ between the CNT and ground plane, namely for $k \gg 1$ has been proposed in [14]

$V_{\mathrm{PI}}=0.85 \sqrt{\frac{1+K^{F K}}{1+K^{T I P}}} \frac{H}{L^{2}} \ln \left(2 \frac{H}{R}\right) \sqrt{\frac{E I}{\varepsilon_{0}}}$,

where the parameters

$$
\begin{aligned}
& K^{F K}=\frac{8 H^{2}}{9 L^{2}}=\frac{8}{9} k^{2} \rho^{2}, \\
& K^{T I P}=2.55 \frac{R^{1 / 3}(H+R)^{2 / 3}}{L}=2.55 \rho(k+1)^{2 / 3},
\end{aligned}
$$

take into account for the effects of finite kinematics and concentrated-tip charge, respectively.Considering the definition (4) $)_{1}$ of the normalized pull-in voltage, from (46) and (47) it follows

\begin{tabular}{|l|ll|}
\hline Journal : Medium 11012 & Dispatch : 9-1-2020 & Pages : 17 \\
Article No. : $\mathbf{1 1 1 9}$ & $\square$ LE & $\square$ TYPESET \\
MS Code : MECC-D-19-00479R2 & $\checkmark_{\mathrm{CP}}$ & $\checkmark$ DISK \\
\hline
\end{tabular}


Table 1 Lower and upper bounds of the pull-in parameters for a CNT switch with $k=1.0$, for various values of the van der Waals parameter $\gamma$ and geometric ratio $\rho$

\begin{tabular}{|c|c|c|c|c|c|c|c|c|c|c|c|c|}
\hline \multirow{2}{*}{$\begin{array}{l}k=1 \\
\gamma\end{array}$} & \multicolumn{4}{|c|}{$\rho=0.01$} & \multicolumn{4}{|c|}{$\rho=0.05$} & \multicolumn{4}{|l|}{$\rho=0.1$} \\
\hline & $\delta_{L}$ & $\beta_{L}$ & $\delta_{U}$ & $\beta_{U}$ & $\delta_{L}$ & $\beta_{L}$ & $\delta_{U}$ & $\beta_{U}$ & $\delta_{L}$ & $\beta_{L}$ & $\delta_{U}$ & $\beta_{U}$ \\
\hline 0 & 0.5119 & 5.8346 & 0.5193 & 5.9082 & 0.4884 & 4.8695 & 0.4923 & 4.9062 & 0.4700 & 4.0432 & 0.4722 & 4.0619 \\
\hline 0.1 & 0.4017 & 4.2413 & 0.4088 & 4.3134 & 0.3942 & 3.6010 & 0.3996 & 3.6477 & 0.3874 & 3.0298 & 0.3917 & 3.0601 \\
\hline 0.2 & 0.3477 & 3.0363 & 0.3550 & 3.1098 & 0.3437 & 2.5965 & 0.3500 & 2.6497 & 0.3401 & 2.1983 & 0.3455 & 2.2368 \\
\hline 0.3 & 0.3091 & 1.9975 & 0.3167 & 2.0724 & 0.3070 & 1.7162 & 0.3139 & 1.7744 & 0.3050 & 1.4591 & 0.3113 & 1.5043 \\
\hline 0.4 & 0.2785 & 1.0613 & 0.2862 & 1.1374 & 0.2775 & 0.9150 & 0.2848 & 0.9774 & 0.2766 & 0.7805 & 0.2836 & 0.8313 \\
\hline 0.5 & 0.2527 & 0.1975 & 0.2606 & 0.2744 & 0.2526 & 0.1708 & 0.2603 & 0.2365 & 0.2524 & 0.1460 & 0.2600 & 0.2016 \\
\hline
\end{tabular}

Table 2 Lower and upper bounds of the pull-in parameters for a CNT switch with $k=10$, for various values of the van der Waals parameter $\gamma$ and geometric ratio $\rho$

\begin{tabular}{|c|c|c|c|c|c|c|c|c|c|c|c|c|}
\hline \multirow{2}{*}{$\begin{array}{l}k=10 \\
\gamma\end{array}$} & \multicolumn{4}{|c|}{$\rho=0.01$} & \multicolumn{4}{|c|}{$\rho=0.05$} & \multicolumn{4}{|l|}{$\rho=0.1$} \\
\hline & $\delta_{L}$ & $\beta_{L}$ & $\delta_{U}$ & $\beta_{U}$ & $\delta_{L}$ & $\beta_{L}$ & $\delta_{U}$ & $\beta_{U}$ & $\delta_{L}$ & $\beta_{L}$ & $\delta_{U}$ & $\beta_{U}$ \\
\hline 0 & 0.4978 & 18.515 & 0.5029 & 18.687 & 0.4565 & 11.939 & 0.4578 & 11.975 & 0.4363 & 8.2986 & 0.4368 & 8.3090 \\
\hline $2 \times 10^{4}$ & 0.4176 & 14.924 & 0.4235 & 15.114 & 0.3985 & 9.8578 & 0.4015 & 9.9198 & 0.3876 & 6.9270 & 0.3895 & 6.9538 \\
\hline $4 \times 10^{4}$ & 0.3725 & 12.060 & 0.3789 & 12.263 & 0.3607 & 8.0684 & 0.3648 & 8.1521 & 0.3537 & 5.7083 & 0.3567 & 5.7512 \\
\hline $6 \times 10^{4}$ & 0.3394 & 9.5547 & 0.3461 & 9.7693 & 0.3316 & 6.4495 & 0.3365 & 6.5520 & 0.3269 & 4.5860 & 0.3309 & 4.6443 \\
\hline $8 \times 10^{4}$ & 0.3127 & 7.2818 & 0.3197 & 7.5054 & 0.3075 & 4.9490 & 0.3132 & 5.0683 & 0.3043 & 3.5334 & 0.3092 & 3.6063 \\
\hline $10 \times 10^{4}$ & 0.2901 & 5.1768 & 0.2973 & 5.4081 & 0.2868 & 3.5381 & 0.2930 & 3.6725 & 0.2847 & 2.5347 & 0.2904 & 2.6215 \\
\hline
\end{tabular}

Fig. 2 Relations between electrostatic loading parameter $\beta$ and tip deflection $\delta$ obtained from the shooting method, for various geometric ratios $k$ and two different values of $\gamma$. Lower and upper estimates of the pull-in parameters are denoted by small circles and small points, respectively (a)

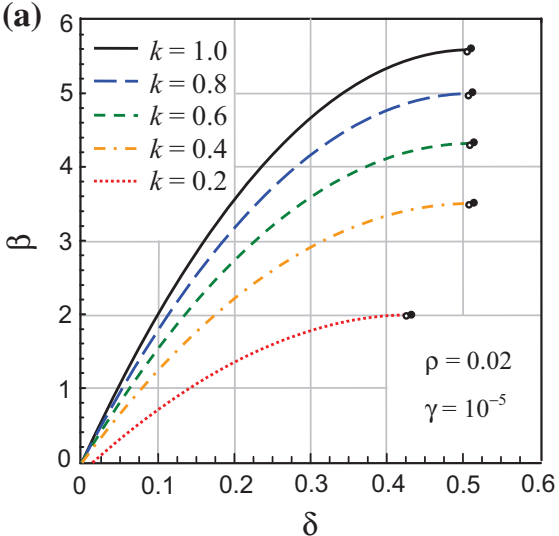

(b)

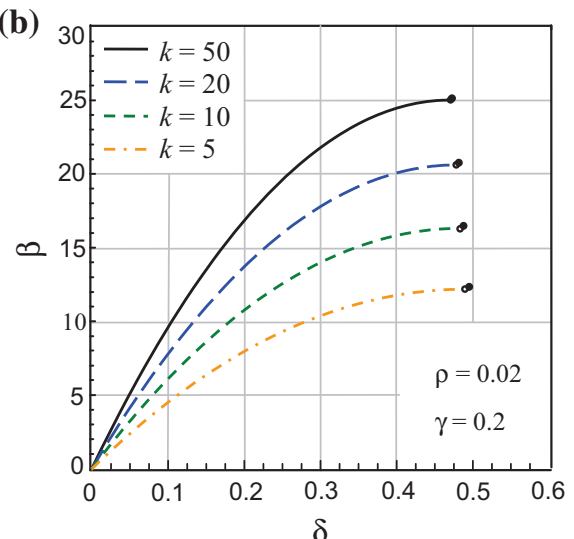

$$
\begin{aligned}
\beta_{\mathrm{Ke}} & =\frac{\pi \varepsilon_{0} V_{P I}^{2} L^{4}}{E I H^{2}}=\pi 0.85^{2} \frac{1+K^{F K}}{1+K^{T I P}} \ln ^{2}\left(2 \frac{H}{R}\right) \\
& =\pi 0.85^{2} \frac{\left(1+8 k^{2} \rho^{2} / 9\right) \ln ^{2}(2 k)}{1+2.55 \rho(k+1)^{2 / 3}} .
\end{aligned}
$$

\section{Results}

Lower and upper estimates for the normalized pull-in voltage $\beta_{L}$ and $\beta_{U}$ and the corresponding estimates of the normalized pull-in deflection $\delta_{U}$ and $\delta_{L}$ have been reported in Tables 1 and 2. In these tables, two different values of the geometric ratio $k$ are considered

\begin{tabular}{|l|ll|}
\hline Journal : Medium 11012 & Dispatch : 9-1-2020 & Pages : 17 \\
Article No. : $\mathbf{1 1 1 9}$ & $\square$ LE & $\square$ TYPESET \\
MS Code : MECC-D-19-00479R2 & $\checkmark_{\mathrm{CP}}$ & $\checkmark$ DISK \\
\hline
\end{tabular}




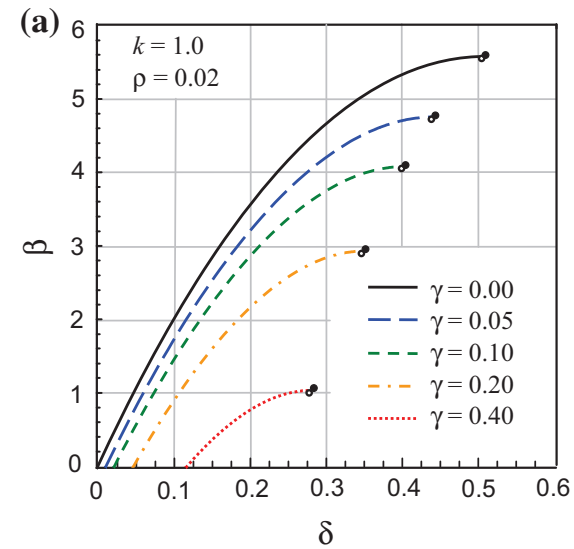

Fig. 3 Relations between electrostatic loading parameter $\beta$ and tip deflection $\delta$ obtained from the shooting method, for the geometric ratios $k=1$ (a) and $k=10$ (b) and various values of

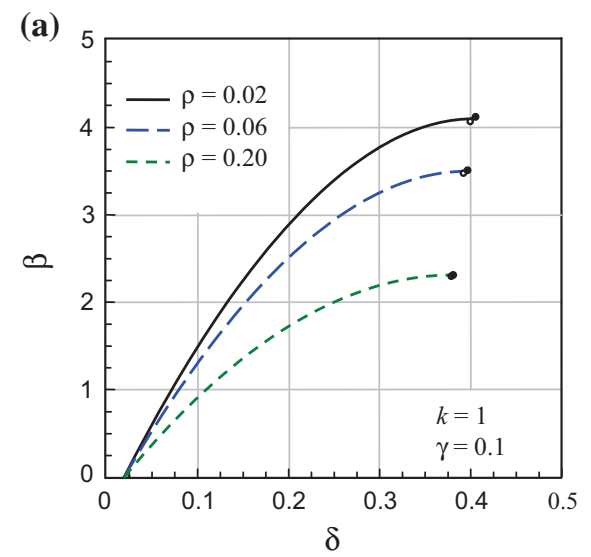

Fig. 4 Relations between electrostatic loading parameter $\beta$ and tip deflection $\delta$ obtained from the shooting method, for $k=1$ and $\gamma=0.1$ (a) and $k=10$ and $\gamma=0.2$ (b), for various

465

466

467

468

469

470

471

472

473

474

475

476

477

478 and results are listed for three specific values of the ratio $\rho=R / L$, which denotes the inverse of the CNT slenderness, and for some specific set of the normalized van der Waals parameter $\gamma$ defined in Eq. (4) 2 .

In order to validate the analytical estimates provided here, the solution to the nonlinear BVP defined by Eqs. (1) and (5) has been calculated by using the numerical integration scheme available in Mathematica $^{\circledR}$, which is based on the shooting method. Figures 2, 3, and 4 show the relationships between the electrostatic loading parameter $\beta$ and tip deflection of the CNT, $\delta=u(1)$, obtained by using the function DSolve of Mathematica ${ }^{\circledR}$, varying the geometric and material parameters of the CNT switch. In these (b)

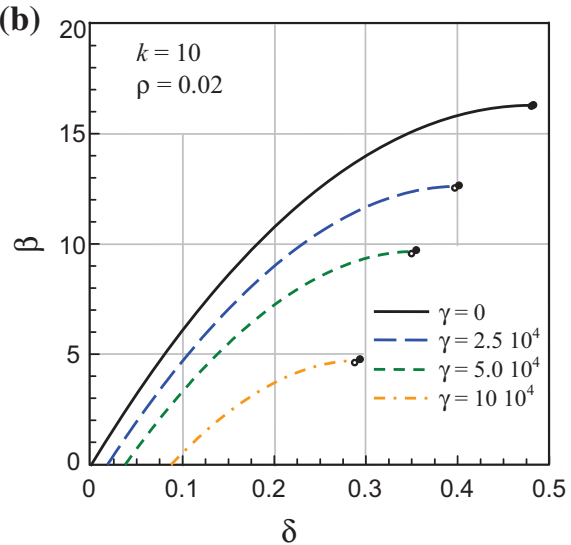

the van der Waals parameter $\gamma$. Lower and upper estimates of the pull-in parameters are denoted by small circles and small points, respectively

(b)

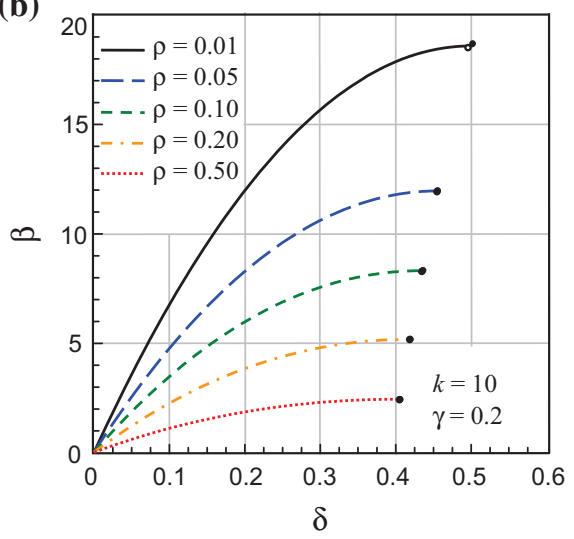

geometric ratios $\rho$. Lower and upper estimates of the pull-in parameters are denoted by small circles and small points, respectively

figures, the lower and upper analytical estimates of the pull-in parameters $\beta_{P I}$ and $\delta_{P I}$ calculated by using the accurate method described in Sects. 4.1 and 4.2 are marked with small circles and points, respectively. In particular, the curves in Fig. 2 display the variation of normalized CNT tip deflection $\delta$ with the electrostatic loading parameter $\beta$ obtained from the shooting method, for various values of the geometric ratio $k$. A slender CNT $(\rho=0.02)$ subject to weak intermolecular surface forces $\left(\gamma=10^{-5} \div 0.2\right)$ is considered therein. These results confirm that the lower and upper analytical bounds for $\beta$ and $\delta$ are very close each other (for all the values of the parameter $k$ considered here), thus ensuring extremely accurate estimates of

\begin{tabular}{|l|lll|}
\hline \multirow{2}{*}{} & Journal : Medium 11012 & Dispatch : 9-1-2020 & Pages : 17 \\
Article No. : 1119 & $\square$ LE & $\square$ TYPESET \\
& MS Code : MECC-D-19-00479R2 & $\checkmark_{\mathrm{CP}}$ & $\checkmark$ DISK \\
\hline
\end{tabular}


the exact pull-in parameters $\beta_{P I}$ and $\delta_{P I}$, which correspond to the maximum of the curves $\beta$ versus $\delta$ obtained by the numerical integration procedure. As expected, the pull-in voltage $\beta_{P I}$ is found to increase with the gap spacing $H$ between the electrodes, which is proportional to the parameter $k$. The pull-in tip displacement $\delta_{P I}$ displays a non monotonic behavior as $k$ is increased. Indeed, it grows for small values of $k$ and then it decreases as $k$ becomes larger. The contribution of the charge concentrated at the CNT free end has been neglected in most investigations, which thus overestimate the pull-in voltage. Actually, the pull-in voltage is significantly reduced when the contribution of the concentrated load acting at the free end is taken into account.

Figure 3 is similar to Fig. 2 except that it focuses on the effects of the van der Waals attractions on the pullin parameters. The same geometric ratio $\rho=0.02$ considered in Fig. 2 has been assumed here. As the beam deflection increases and the normalized gap spacing 1- $u$ decreases, the van der Waals interaction becomes stronger than the electrostatic force. Their magnitude indeed varies with the gap spacing according to the different laws introduced in (3). If the magnitude $\gamma$ of the van der Waals interaction increases, then it becomes effective at larger gap spacing and, thus, both the pull-in voltage and the pullin tip deflection are found to decrease, as it can be observed in Fig. 3a, b. These plots also confirm that the analytical lower and upper bounds for $\beta$ and $\delta$ are very close each other and, thus, also to the exact pullin parameters $\beta_{P I}$ and $\delta_{P I}$, which should lay in between.
The effects of the geometric ratio $\rho$ on the pull-in parameters can be observed in Fig. 4 for two sets of values of $\gamma$ and $k$. As $\rho$ decreases, namely for slender CNT, the normalized pull-in voltage $\beta_{P I}$ increases together with the corresponding normalized tip deflection $\delta_{P I}$. Note the effects of the CNT slenderness ratio $\rho$ are more evident for large gap spacing, namely for $k \gg 1$ (Fig. 4b).

The variations of the van der Waals parameters $\gamma$ with the tip displacement $\delta$ for a freestanding CNT cantilever $(\beta=0)$ obtained by numerical integration are plotted in Fig. 5 for various values of the geometric ratio $k$. If the parameter $\gamma$ exceeds its critical value $\gamma_{P I}$, which is given by the maximum of the $\gamma-\delta$ curve obtained by numerical integration, then pull-in instability occurs even if no electric voltage is applied to the electrodes. It can be observed that the estimated values of $\gamma_{P I}$ and the corresponding pull-in deflection $\delta_{P I}$ agree very well with the results of the numerical procedure. These plots also show that the critical values of van der Waals parameter is increased by increasing the geometric ratio $k$. No significant influence of $k$ has been observed on the normalized pull-in tip deflection $\delta_{P I}$, which turns out to be about constant and equal to 0.25 , independently of $k$. Lower and upper estimates of critical van der Waals parameter $\gamma_{P I}$ and tip deflection $\delta_{P I}$ for a freestanding CNT can be found in Table 3 for some values of the geometric ratio $k$. There, it can be noted that a stronger van der Waals force is required to induce the pull-in instability as the normalized gap spacing $k$ increases, whereas the normalized pull-in tip deflection $\delta$ is almost independent of $k$. Note that the geometric ratio $\rho$ has no effect on the pull-in value $\gamma_{P I}$ of the van der
Fig. 5 Relations between van der Waals parameter $\gamma$ and tip deflection $\delta$ obtained from the shooting method, for a freestanding nanotube $(\beta=0)$ and for small (a) and large (b) values of the geometric ratio $k$. Lower and upper estimates of the pullin parameters are denoted by small circles and small points, respectively (a)

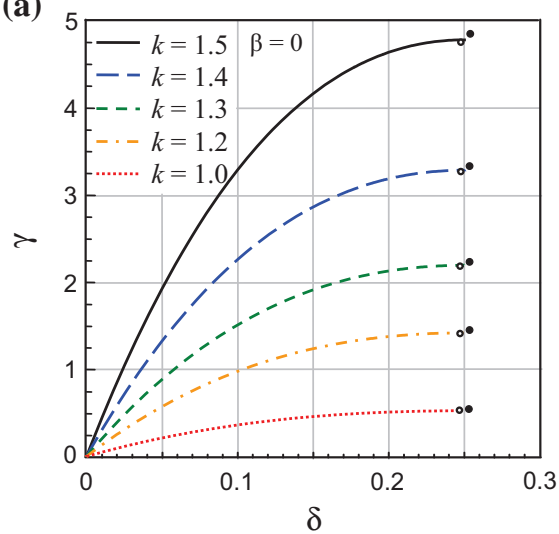

(b)

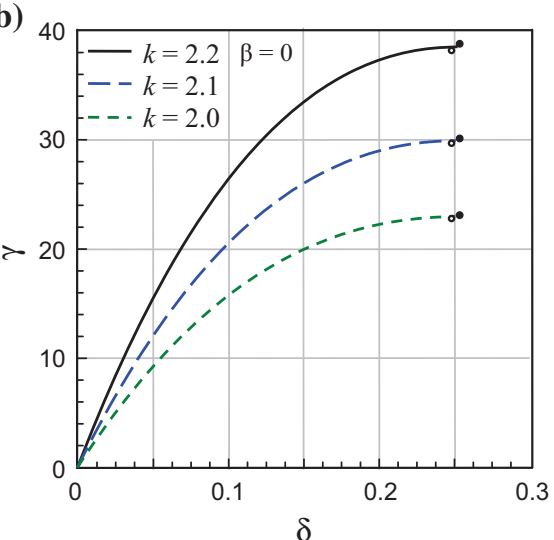


Meccanica

Table 3 Lower and upper bounds for the parameters $\gamma$ and $\delta$ causing the pull-in instability in the absence of electrostatic actuation $(\beta=0)$ and approximated value $\gamma_{P I}^{*}$ provided by Eq. (49), for various values of the geometric ratio $k$

\begin{tabular}{llllll}
\hline$k$ & $\gamma_{P I}^{*}$ & $\gamma_{L}$ & $\delta_{L}$ & $\gamma_{U}$ & $\delta_{U}$ \\
\hline 0.2 & $7.16 \times 10^{-5}$ & $7.72 \times 10^{-5}$ & 0.2456 & $7.86 \times 10^{-5}$ & 0.2513 \\
0.4 & $3.24 \times 10^{-3}$ & $3.47 \times 10^{-3}$ & 0.2459 & $3.53 \times 10^{-3}$ & 0.2517 \\
0.6 & $3.01 \times 10^{-2}$ & $3.21 \times 10^{-2}$ & 0.2463 & $3.26 \times 10^{-2}$ & 0.2521 \\
0.8 & $1.47 \times 10^{-1}$ & $1.55 \times 10^{-1}$ & 0.2467 & $1.58 \times 10^{-1}$ & 0.2525 \\
1 & $5.00 \times 10^{-1}$ & $5.24 \times 10^{-1}$ & 0.2471 & $5.33 \times 10^{-1}$ & 0.2529 \\
2 & $2.26 \times 10^{1}$ & $2.28 \times 10^{1}$ & 0.2485 & $2.32 \times 10^{1}$ & 0.2543 \\
3 & $2.10 \times 10^{2}$ & $2.06 \times 10^{2}$ & 0.2485 & $2.09 \times 10^{2}$ & 0.2543 \\
4 & $1.02 \times 10^{3}$ & $9.83 \times 10^{2}$ & 0.2477 & $1.00 \times 10^{3}$ & 0.2535 \\
5 & $3.49 \times 10^{3}$ & $3.32 \times 10^{3}$ & 0.2465 & $3.38 \times 10^{3}$ & 0.2523 \\
10 & $1.58 \times 10^{5}$ & $1.55 \times 10^{5}$ & 0.2406 & $1.58 \times 10^{5}$ & 0.2463 \\
20 & $7.15 \times 10^{6}$ & $7.99 \times 10^{6}$ & 0.2345 & $8.14 \times 10^{6}$ & 0.2401 \\
50 & $1.10 \times 10^{9}$ & $1.67 \times 10^{9}$ & 0.2294 & $1.70 \times 10^{9}$ & 0.2348 \\
\hline
\end{tabular}

Fig. 6 Variation of the van der Waals parameter $\gamma_{P I}^{*}$ with the geometric ratio $k$. The upper and lover values of $\gamma$ causing the pullin instability for a freestanding CNT cantilever are marked by full and empty circles, respectively

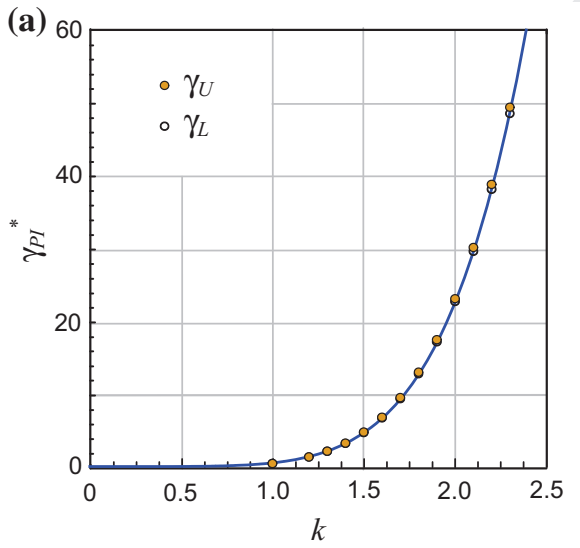

Fig. 7 Normalized variations of the pull-in voltage $\beta$ with the van der Waals parameter $\gamma$, for small (a) and large (b) values of the geometric ratios $k$

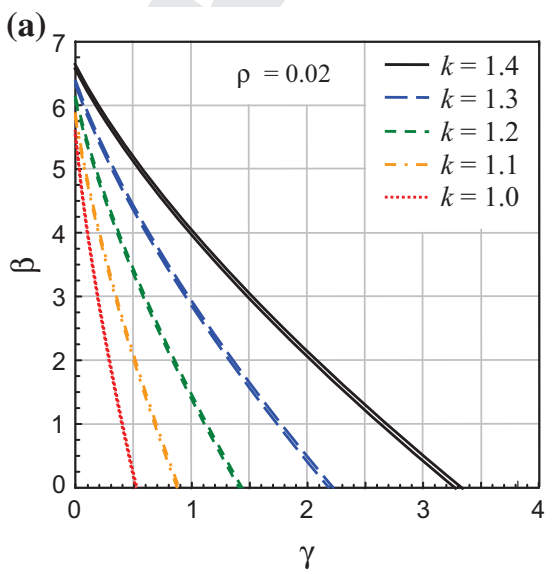

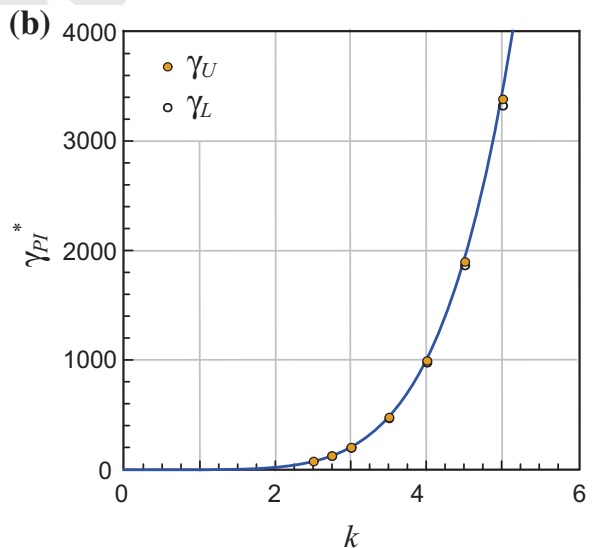

(b)

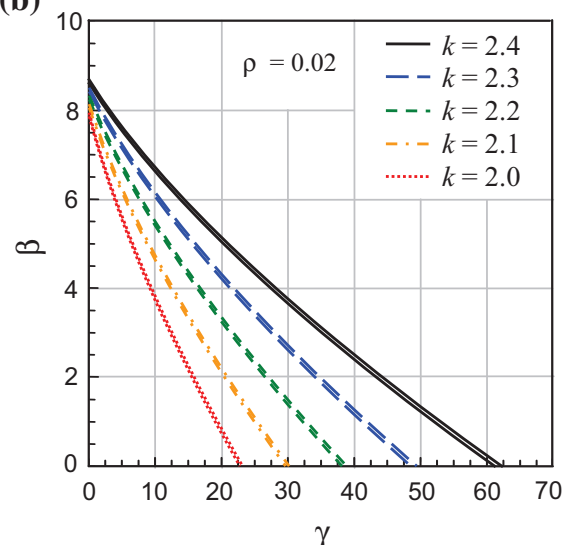

Waals parameter. Indeed, according to Eq. (6) $\rho$

561 affects the concentrated tip load only, which is

562 vanishing for $\beta=0$.
On the basis of the performed investigations a 563 simple closed-form relation is proposed here for the

\begin{tabular}{|l|ll|} 
Journal : Medium 11012 & Dispatch : 9-1-2020 & Pages : 17 \\
Article No. : 1119 & $\square$ LE & $\square$ TYPESET \\
MS Code : MECC-D-19-00479R2 & $\checkmark$ CP & $\checkmark$ DISK \\
\hline
\end{tabular}


pull-in value of the van der Waals parameter for a

$$
\gamma_{P I}^{*}=\frac{1}{2} k^{11 / 2} .
$$

The variations of $\gamma_{P I}^{*}$ with $k$ are plotted in Fig. 6a, b together with the upper and lower bounds $\gamma_{U}$ and $\gamma_{L}$ provided by the present analysis. Values of $\gamma_{P I}^{*}$ for some specific value of $k$ have been reported in Table 3 also. In Fig. 6a, it can be noted that relation (49) fits very well the lower bounds $\gamma_{U}$ for small values of $k$, namely for $k<2.8$, and thus it can be conveniently used for the safe design of CNT switches with a small gap spacing. Equation (49) provides accurate predictions also for $k>2.8$, as it can be observed in Fig. 6b, but in this case $\gamma_{P I}^{*}$ may result a bit larger than $\gamma_{U}$, as it can be noted in Table 3 for $k=3 \div 5$. Relation (49) actually defines a minimum gap spacing $H_{\min }$ or, equivalently, a maximum CNT length $L_{\max }$ for preventing the pull-in collapse of a CNT in the absence of electrostatic loading, namely

$$
\begin{gathered}
H_{\min }=\left(C_{6} \sigma^{2} \frac{\pi^{2} L^{4}}{E I}\right)^{2 / 11} R^{1 / 11}, \\
L_{\max }=\left(\frac{E I}{C_{6} \sigma^{2} \pi^{2}}\right)^{1 / 4}\left(\frac{H^{11}}{R}\right)^{1 / 8} .
\end{gathered}
$$

The variations of $\beta_{U}$ and $\beta_{L}$ with $\gamma$ are plotted in Fig. 7 for various value of the geometric ratio $k$. These estimates are very close each other and, thus, extremely accurate, for every value of the van der Waals parameter. Both the pull-in voltage $\beta$ and the limit value of the coefficients $\gamma$ increase as the parameter $k$ is increased. In general, for assigned geometry, namely for given values of $\rho$ and $k$, the pullin voltage decreases as the strength of the van der Waals attractions increases. The pull-in voltage
Fig. 8 Variations of lower and upper bounds $\beta_{L}$ and $\beta_{U}$ of the pull-in voltage with the geometric ratio $k$, for $\gamma=0$ and for various geometric ratios $\rho$ (a)

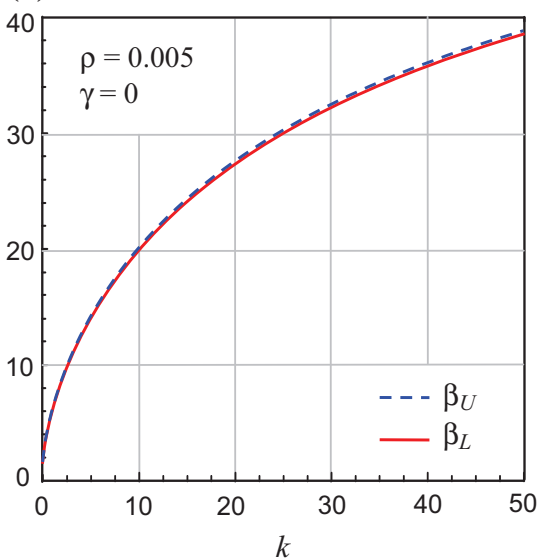

(c)

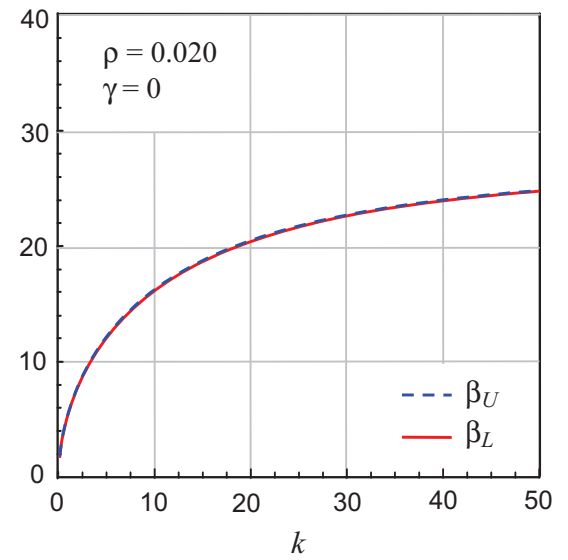

(b)

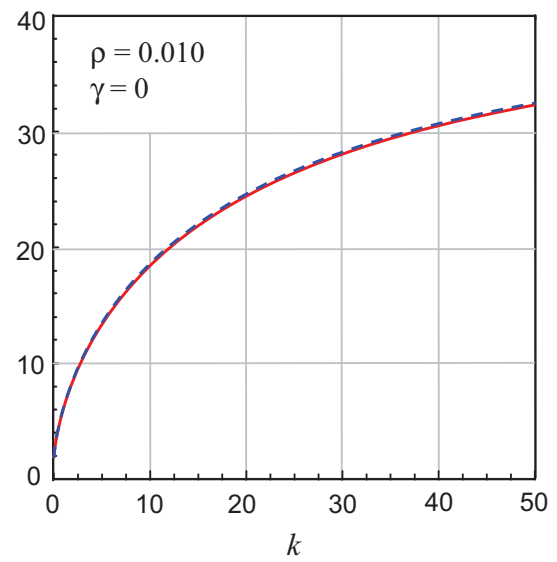

(d)

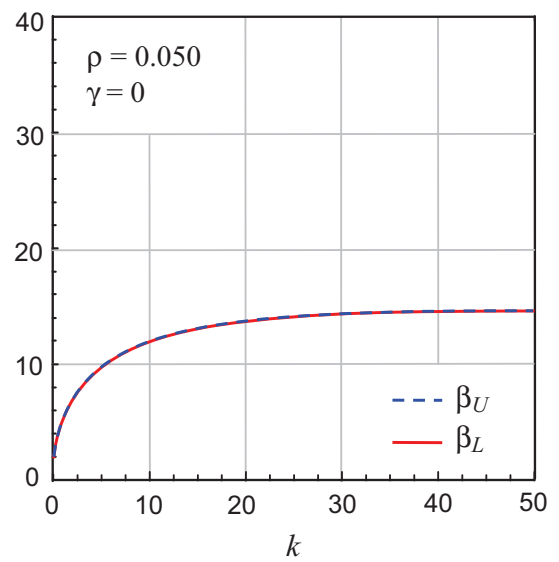

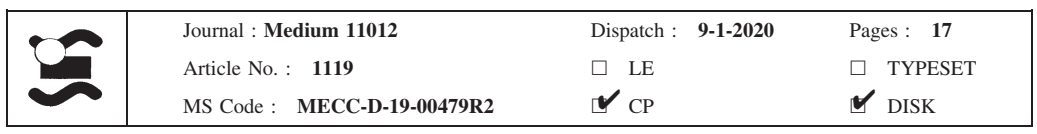


Fig. 9 Variations of $\delta_{L}$ and $\delta_{U}$ with the geometric ratio $k$, for $\gamma=0$ and for two different geometric ratios $\rho$

Fig. 10 Variations of $\beta_{L}$ and $\beta_{U}(\mathbf{a})$, and $\delta_{L}$ and $\delta_{U}$ (b) with the geometric ratio $\rho$ for two different values of the geometric ratio $k$ and $\gamma=0$ (a)

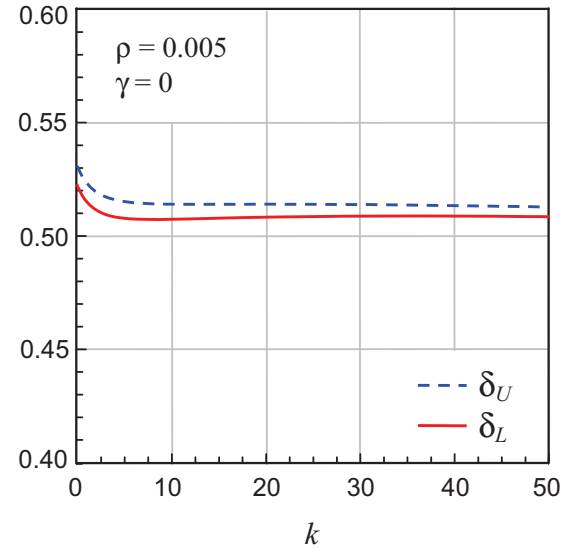

(a)

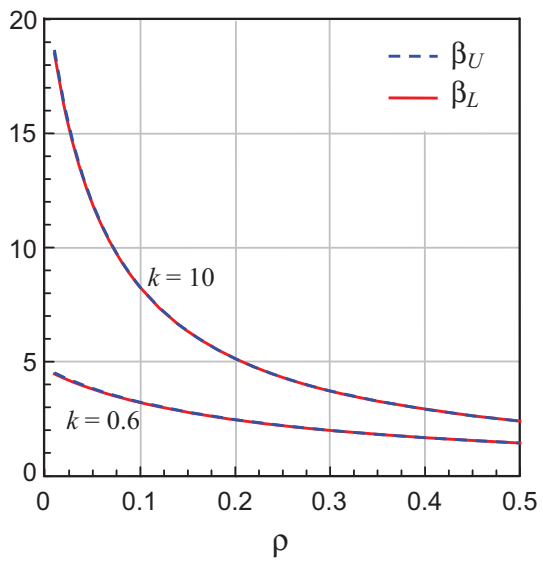

(b)

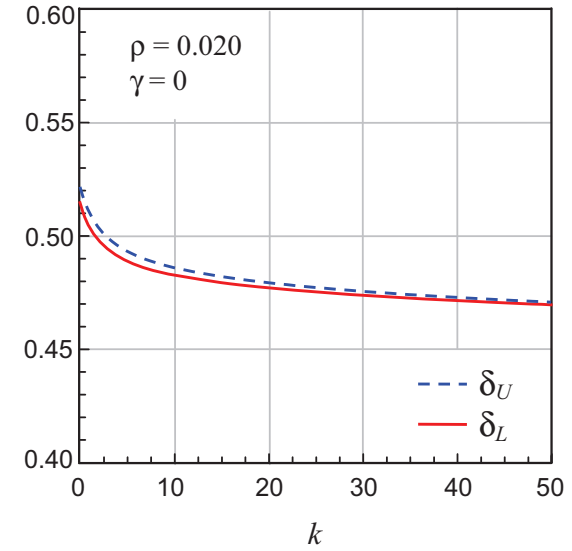

(b)

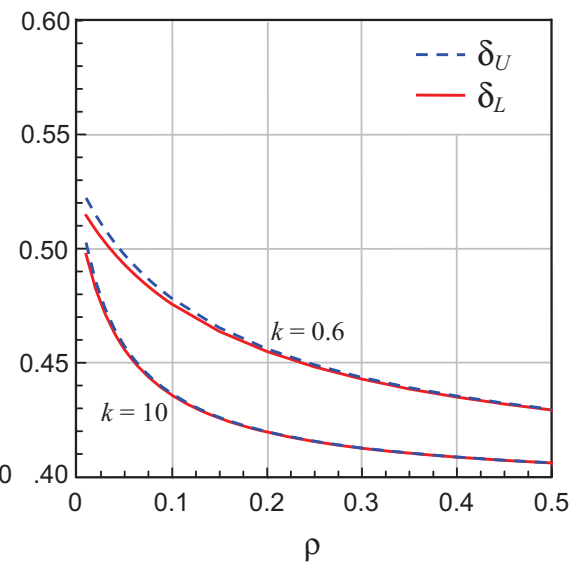

vanishes when the van der Waals parameter attains its critical values $\gamma_{\mathrm{PI}}$. Negative values of $\beta$ then imply that a repulsive electrostatic force is required to prevent pull-in instability induced by the van der Waals attraction when it overcomes the elastic restoring force. In this case, the CNT collapses onto and adheres to the ground plane in the absence of electrostatic actuation, due only to the van der Waals attraction that is responsible of the occurring of stiction [31]. This phenomenon is exploited in non-volatile memory cells, where the switch is hold in the closed state with no need of continued power input. The occurrence of stiction in applications such as nanoactuators, nanoresonators and nano-tweezers may instead limit the range of operability of the device and lead to undesirable consequences.

The variations of lower and upper bounds of the pull-in parameters $\beta_{P I}$ and $\delta_{P I}$ with the geometric ratio $k$ are plotted in Figs. 8 and 9, respectively, for vanishing contribution of the van der Waals force $(\gamma=0)$. The lower and upper analytical bounds turn out to be very close each other for every value of the geometric ratio $k$, thus ensuring accurate estimates of the pull-in parameters. Moreover, the pull-in voltage is found to increase with the gap spacing parameter $k$, as expected, and it seems to approach an almost constant limit values for large $k$. Note that the pull-in deflection $\delta_{P I}$ display a limited variation with $k$ so that the range of variation of the plots in Fig. 9 has been restricted between 0.4 and 0.6 to make the gap more visible. Due to the adopted graphical representation, it may seem that the predicted upper and lower pull-in deflections $\delta_{L}$ and $\delta_{U}$ in Fig. 9 are more separated than the upper and lower pull-in voltages plotted in Fig. 8, but actually the former are as close as the latter.

\begin{tabular}{|l|lll|}
\hline \multirow{2}{*}{ Journal : Medium 11012 } & Dispatch : 9-1-2020 & Pages : 17 \\
Article No. : 1119 & $\square$ LE & $\square$ TYPESET \\
MS Code : MECC-D-19-00479R2 & $\checkmark$ CP & $\checkmark$ DISK \\
\hline
\end{tabular}


Fig. 11 Variation of approximated normalized pull-in voltage $\beta_{\mathrm{Ke}}$ with $k$ for four values of slenderness ratio $\rho$ : considering both effects of concentrated charge and finite kinematics (solid line), considering only the effect of concentrated charge (dashed line), neglecting both effects (dash-dotted line). The analytical predictions of the lower and upper bounds are plotted by solid and dashed black lines, respectively
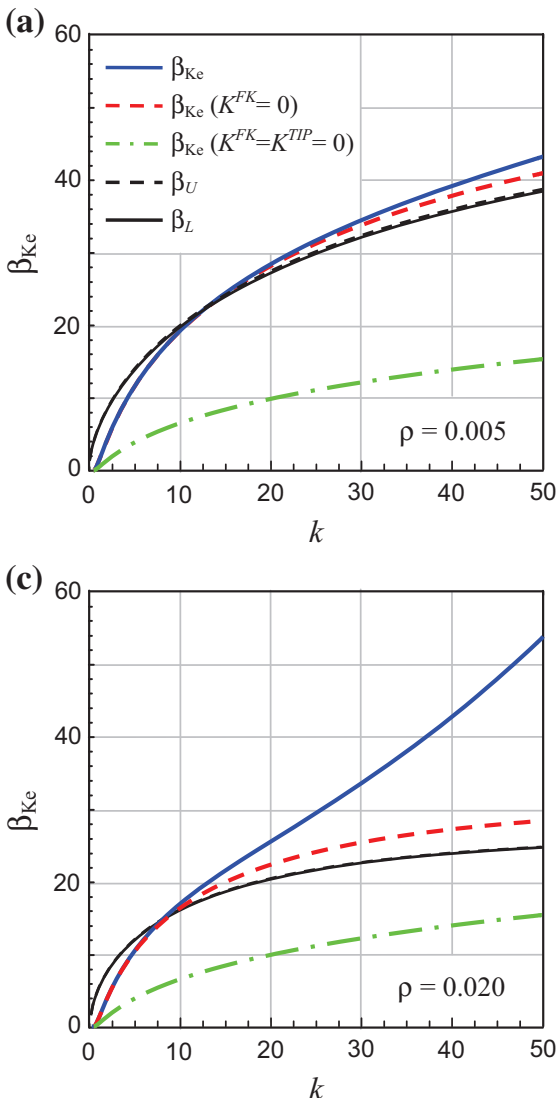

(b)

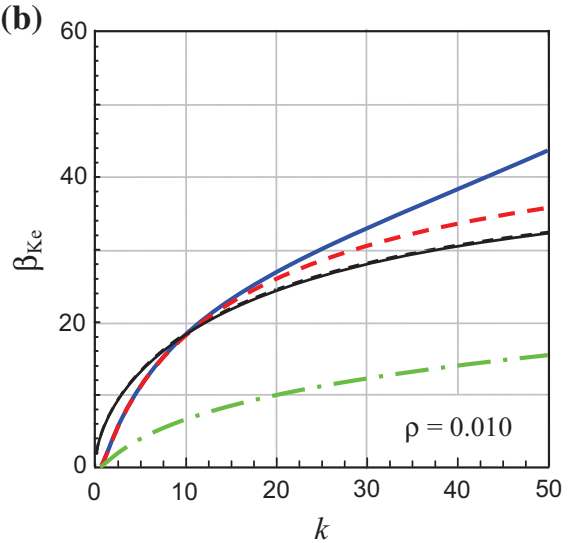

(d)

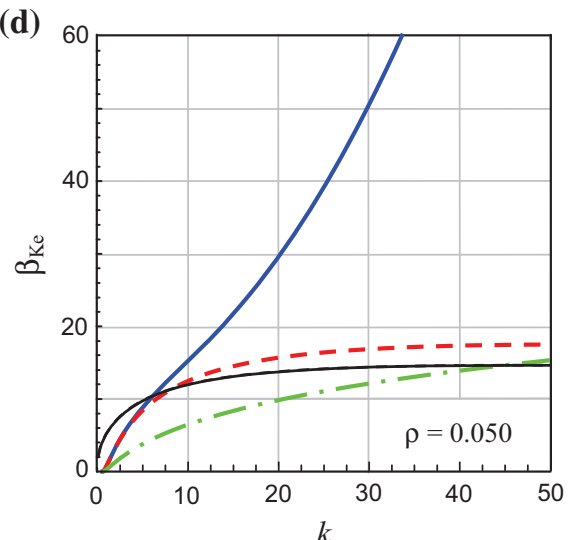

The variations of lower and upper bounds of the pull-in parameters $\beta_{P I}$ and $\delta_{P I}$ with the geometric ratio $\rho$ are plotted in Fig. 10a, b, respectively, neglecting the contribution of the van der Waals attractions $(\gamma=0)$. It can be observed that increasing the geometric ratio $\rho$ results in decreasing the pull-in voltage (Fig. 10a) and the normalized pull-in displacement (Fig. 10b). The rapid variation observed for $k=10$, namely for large gap spacing, proves that the pull-in parameters are very sensitive to the geometric ratio $\rho$, especially when it is small, namely for very slender CNTs. Note the reduced range of variation considered for $\delta_{L}$ and $\delta_{U}$ in Fig. 10b.

According to Eq. (48), the variations of the approximated pull-in voltage $\beta_{\mathrm{Ke}}$ proposed by Ke et al. [14] with $k$ are plotted in Fig. 11 for four values of $\rho$. In particular, the blue solid lines take into consideration both the effects of concentrated charge and finite kinematics, the red dashed lines take into consideration the effect of concentrated charge only, and the green dash-dotted lines neglect both effects. The analytical predictions of the lower and upper bounds proposed here are plotted in the same figures by solid and dashed black lines, respectively. From Fig. 11 it can be observed that the effect of finite kinematics, namely the term $K^{F K}$, is negligible for $k<20$ if $\rho=0.005$, for $k<15$ if $\rho=0.01$, for $k<10$ if $\rho=0.02$, and for $k<5$ if $\rho=0.05$, whereas the effect of concentrated charge $K^{T I P}$ can never be neglected. Moreover, if the effects of finite kinematics are neglected, relation (48) roughly approximates the estimates of the pull-in voltage obtained by the present approach. However, Eq. (48) provides estimates of the pull-in voltage smaller than the lower bound $\beta_{L}$ for small values of $k$ and larger than the upper bound $\beta_{U}$ for large values of $k$.

\section{Conclusions}

Analytical lower and upper bounds for the pull-in voltage and deflection of an electro-statically actuated 667

651

652

653

654

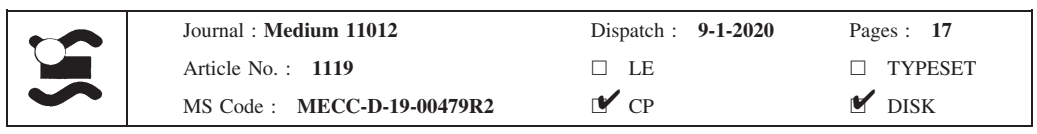




$$
\begin{aligned}
& h(0)=0, \quad h(1)=0, \quad h^{\prime}(0)=0, \quad h^{\prime \prime}(1)=0, \\
& h^{\prime \prime \prime}(1)=0,
\end{aligned}
$$

704 and

$$
h^{\mathrm{V}}(x) \leq 0, \quad \text { for } \quad x \in[0,1]
$$

CNT cantilever switch are proposed and then valiated by comparison with the results obtained from a erical integration procedure of the governing for every set of material and loading parameter considered here, thus proving the efficiency of the proposed approach. Moreover, they are found to improve the accuracy with respect to approximated lations proposed in the literature for the fast estimate pull-in voltage of CNT switches.

conclusion, the present study can be regarded as during operation, thus assuring robust and consistent Acknowledgements Support from the Italian "Gruppo Nazionale di Fisica Matematica" INdAM-GNFM is gratefully

Conflict of interest The authors declare that they have no conflict of interest.

\section{then}

$h(x) \geq 0, \quad$ for $\quad x \in[0,1]$

Proof By using the mean value theorem, from continuity and conditions $(51)_{1,2}$ it follows that there exists $x_{1} \in[0,1]$ such that $h^{\prime}\left(x_{1}\right)=0$. Then, by using conditions $(51)_{3,4}$ there exist $x_{2} \in\left[0, x_{1}\right]$ and $x_{3} \in\left[x_{2}\right.$, 1] such that $h^{\prime \prime}\left(x_{2}\right)=0$ and $h^{\prime \prime \prime}\left(x_{3}\right)=0$. Since the function $h^{\prime \prime \prime}(x)$ is concave for $x \in[0,1]$ according to (52), it follows that $h^{\prime \prime}(x) \leq 0$ for $x \in\left[x_{2}, 1\right]$ and $h^{\prime \prime}(x) \geq 0$ for $x \in\left[0, x_{2}\right]$. Therefore, $h^{\prime}(x) \geq 0$ for $x \in\left[0, x_{1}\right]$ and $h^{\prime}(x) \leq 0$ for $x \in\left[x_{1}, 1\right]$. Since $h(0)=h(1)=0$ according to Eq. $(51)_{1,2}$, then it necessarily follows that $h(x) \geq 0$ for $x \in[0,1]$, so that condition (53) holds true.

Lemma B Let the function $g(x)$ be continuous up to the third derivative for $x \in[0,1]$ and satisfy the following conditions

$g(0)=0, \quad g(1)=0, \quad g^{\prime}(0)=0, \quad g^{\prime \prime}(1)=0$.

and

$g^{\mathrm{IV}}(x) \geq 0, \quad$ for $\quad x \in[0,1]$

then

$g(x) \geq 0, \quad$ for $\quad x \in[0,1]$

Proof By using the mean value theorem, from conditions $(54)_{1,2}$ it follows that there exists $x_{1} \in[0,1]$ such that $g^{\prime}\left(x_{1}\right)=0$. Moreover, by using conditions $(54)_{3,4}$ there exists $x_{2} \in\left[0, x_{1}\right]$ such that $g^{\prime \prime}\left(x_{2}\right)=0$. Condition (55) then implies that $g^{\prime \prime}(x)$ is convex. It follows that $g^{\prime \prime}(x) \leq 0$ for $x \in\left[x_{2}, 1\right]$ and $g^{\prime \prime}(x) \geq 0$ for $x \in\left[0, x_{2}\right]$, and thus $g^{\prime}(x) \geq 0$ for $x \in\left[0, x_{1}\right]$ and $g^{\prime}(x) \leq 0$ for $x \in\left[x_{1}, 1\right]$. Since $g(0)=g(1)=0$ according to conditions (54) 1,2 , then it necessarily follows that inequality (56) holds true.

\section{References}

1. Bogue R (2009) Nanosensors: a review of recent research. $\mathbf{A Q 3} 740$ Sens Rev 29(4):310-315

2. Kim P, Lieber CM (1999) Nanotube nanotweezers. Science 742 286:2148-2150 743

3. Akita S, Nakayama Y, Mizooka S, Takano Y, Okawa T, 744 Miyatake Y, Yamanaka S, Tsuji M, Nosaka T (2001) 745 Nanotweezers consisting of carbon nanotubes operating in 746 an atomic force microscope. Appl Phys Lett 79:1691-1693 747

\begin{tabular}{|l|lll|}
\hline \multirow{2}{*}{0} & Journal : Medium 11012 & Dispatch : 9-1-2020 & Pages : 17 \\
& Article No. : 1119 & $\square$ LE & $\square$ TYPESET \\
& MS Code : MECC-D-19-00479R2 & $\checkmark$ CP & $\checkmark$ DISK \\
\hline
\end{tabular}


4. Dequesnes M, Rotkin SV, Aluru NR (2002) Calculation of pull-in voltages for carbon-nanotube-based nanoelectromechanical switches. Nanotechnol 13:120-131

5. Wang GW, Zhang Y, Zhao YP, Yang GT (2004) Pull-in instability study of carbon nanotube tweezers under the influence of van der Waals forces. J Micromech Microeng 14:1119-1125

6. Ouakad HM, Younis MI (2010) Nonlinear dynamics of electrically actuated carbon nanotube resonators. J Comp Nonlinear Dyn 5(1):011009

7. Bornassi S, Haddadpour H (2017) Nonlocal vibration and pull-in instability analysis of electrostatic carbon-nanotube based NEMS devices. Sens Actuators A Phys 266:185-196

8. Sedighi HM, Farjam N (2017) A modified model for dynamic instability of CNT based actuators by considering rippling deformation, tip-charge concentration and Casimir attraction. Microsyst Technol 23(6):2175-2191

9. Mukherjee B, Sen S (2018) Generalized closed form solutions for feasible dimension limit and pull-in characteristics of nanocantilever under the influences of van der Waals and Casimir forces. Mater Res Express 5(4):045028

10. Farokhi H, Païdoussis MP, Misra AK (2018) Nonlinear behaviour of cantilevered carbon nanotube resonators based on a new nonlinear electrostatic load model. J Sound Vib 419:604-629

11. Mobki H, Rezazadeh G, Vefaghi A, Moradi MV (2019) Investigation of nonlinear dynamic behavior of a capacitive carbon nano-tube based electromechanical switch considering van der Waals force. Microsyst Technol 25(2):461-475

12. Fakhrabadi MMS, Khorasani PK, Rastgoo A, Ahmadian MT (2013) Molecular dynamics simulation of pull-in phenomena in carbon nanotubes with Stone-Wales defects. Solid State Commun 157:38-44

13. Ke CH, Espinosa HD, Pugno N (2005) Numerical analysis of nanotube based NEMS devices-part II: role of finite kinematics, stretching and charge concentrations. J Appl Mech 72(5):726-731

14. Ke CH, Pugno N, Peng B, Espinosa HD (2005) Experiments and modeling of carbon nanotube-based NEMS devices. J Mech Phys Solids 53(6):1314-1333

15. Ke CH (2016) Electromechanical properties and applications of Carbon nanotube nanocantilevers. In: Voiculescu I, Zaghloul M (eds) Nanocantilever beams, modeling, fabrication and applications, pp 195-220

16. Radi E, Bianchi G, di Ruvo L (2017) Upper and lower bounds for the pull-in parameters of a micro- or nanocantilever on a flexible support. Int J Non-Linear Mech 92:176-186

17. Radi E, Bianchi G, di Ruvo L (2018) Analytical bounds for the electro-mechanical buckling of a compressed nanocantilever. Appl Math Model 59:571-572

18. Ke CH, Espinosa HD (2005) Nanoelectromechanical systems and modeling. In: Rieth M, Schommers W (eds) Handbook of theoretical and computational nanotechnology, vol 1, pp 1-38
19. Ke CH, Espinosa HD (2005) Numerical analysis of nanotube-based NEMS devices-part I: electrostatic charge distribution on multiwalled nanotubes. J Appl Mech 72(5):721-725

20. Farrokhabadi A, Abadian N, Rach R, Abadyan M (2014) Theoretical modeling of the Casimir force-induced instability in freestanding nanowires with circular cross-section. Phys E Low Dimens Syst Nanostruct 63:67-80

21. Farrokhabadi A, Abadian N, Kanjouri F, Abadyan M (2014) Casimir force-induced instability in freestanding nanotweezers and nanoactuators made of cylindrical nanowires. Int J Mod Phys B 28(19):1450129

22. Ouakad HM, Sedighi HM (2016) Rippling effect on the structural response of electrostatically actuated single-walled carbon nanotube based NEMS actuators. Int J NonLinear Mech 87:97-108

23. Batra RC, Sears A (2007) Continuum models of multiwalled carbon nanotubes. Int J Solids Struct 44:7577-7596

24. Koochi A, Kazemi AS, Noghrehabadi A, Yekrangi A, Abadyan M (2011) New approach to model the buckling and stable length of multi walled carbon nanotube probes near graphite sheets. Mater Des 32:2949-2955

25. Karimipour I, Kanani A, Koochi A, Keivani M, Abadyan M (2015) Modeling the electromechanical behavior and instability threshold of NEMS bridge in electrolyte considering the size dependency and dispersion forces. Phys E 74:140-150

26. Firouzi B, Zamanian M (2019) The effect of capillary and intermolecular forces on instability of the electrostatically actuated microbeam with T-shaped paddle in the presence of fringing field. Appl Math Model 71:243-268

27. Espinosa HD, Ke CH (2007) Nanoelectromechanical systems-experiments and modeling. In: Bhushan B, Fuchs H (eds) Applied scanning probe methods VII. Nanoscience and technology. Springer, Berlin, pp 135-196

28. Mokhtari J, Farrokhabadi A, Rach R, Abadyan M (2015) Theoretical modeling of the effect of Casimir attraction on the electrostatic instability of nanowire-fabricated actuators. Phys E Low Dimens Syst Nanostruct 68:149-158

29. Sears A, Batra RC (2004) Macro-mechanics properties of carbon nanotubes from molecular mechanics simulations. Phys Rev B 69:235406

30. Duan J, Li Z, Liu J (2016) Pull-in instability analyses for NEMS actuators with quartic shape approximation. Appl Math Mech 37:303-314

31. Loh O, Wei XD, Ke CH, Sullivan J, Espinosa HD (2011) Robust Carbon-nanotube-based-electromechanical devices: understanding and eliminating prevalent failure modes using alternative electrode materials. Small 7(1):79-86

Publisher's Note Springer Nature remains neutral with regard to jurisdictional claims in published maps and institutional affiliations.

\begin{tabular}{|l|lll|}
\hline & Journal : Medium 11012 & Dispatch : 9-1-2020 & Pages : 17 \\
Article No. : 1119 & $\square$ LE & $\square$ TYPESET \\
MS Code : MECC-D-19-00479R2 & $\checkmark$ CP & $\checkmark$ DISK \\
\hline
\end{tabular}


Journal : 11012

Article : 1119

\section{Author Query Form}

\section{Please ensure you fill out your response to the queries raised below and return this form along with your corrections}

Dear Author

During the process of typesetting your article, the following queries have arisen. Please check your typeset proof carefully against the queries listed below and mark the necessary changes either directly on the proof/online grid or in the 'Author's response' area provided below

\begin{tabular}{|l|l|l|}
\hline Query & Details Required & Author's Response \\
\hline AQ1 & $\begin{array}{l}\text { Please check and confirm that the authors and their respective affiliations have been correctly } \\
\text { identified and amend if necessary. }\end{array}$ & \\
\hline AQ2 & Please confirm the corresponding author and corresponding affiliation are correctly identified. & \\
\hline AQ3 & Reference citations have been changed to sequential order in text. Please check and confirm. & \\
\hline AQ4 & Please provide publisher name for the references $[15,18]$. & \\
\hline
\end{tabular}

